\title{
La salud en la seguridad social La reforma de salud en los Estados Unidos: lo bueno, lo malo y lo que podemos aprender*
}

\author{
Healthcare and social security. \\ The reform of healthcare in the United States: its good and bad \\ aspects, and what we can learn thereof \\ Guillermo Vanegas • Oakland, California, EE.UU.
}

Para mí es un gran honor y un especial privilegio el haber recibido el encargo de dictar la Conferencia Lombana Barreneche en este XXI Congreso Colombiano de Medicina Interna, y sobre todo, dirigirme a este auditorio tan selecto, y particularmente en la ciudad de Cali, en este bello centro de convenciones. Me da orgullo, y agradezco a Juan Ramón Abello y a Gregorio Sánchez por haber hecho posible que yo esté aquí, cuando el congreso ha vuelto a Cali después de 20 años.

Muchos de ustedes deben todavía recordar todo el tiempo y el trabajo que le dedicamos juntos, a tratar de influir la gestión y luego la implementación de la Ley 100 en la década de los años 90, y cuántos desvelos y frustraciones, más que logros y satisfacciones, tuvimos en el proceso. Desde hace muchos años he tenido un especial interés por el tema de la salud en la seguridad social, no sólo bajo la perspectiva del médico, su papel en ella y las consecuencias para su práctica profesional, sino bajo la perspectiva de la calidad de la atención que a través de ella reciben los pacientes, que son nuestra razón de ser.

Como todos ustedes bien lo saben y lo practican todos los días, nuestra misión como médicos no es solamente curar la enfermedad y aliviar el dolor, sino mantener la salud y prevenir la enfermedad. Nuestra responsabilidad ante la sociedad va más allá de la atención y el cuidado que le damos a todos, y cada uno de nuestros pacientes e incluye trabajar por el mantenimiento de la salud de la sociedad, de la cual hacemos parte y a la que le debemos lo que somos.

El progreso cada vez más amplio y rápido de la ciencia y la tecnología, ha dado a la medicina las herramientas para prolongar la expectativa de vida y mejorar su calidad. Todos estos beneficios, sin embargo, han venido con una espiral de costos cada vez mayor e inatajable. Ya desde principios del siglo XX se empezó a hacer evidente, que el creciente costo de la atención médica rebasaba la capacidad de pago del ciudadano medio, y no pasó mucho tiempo para que superara también la capacidad de los de mayor fortuna, dando así, por necesidad y oportunidad, origen a la industria de los seguros de salud. Pero ésto era una buena opción para los que podían pagarlos. Para los de bajos recursos solo estaba la caridad, cuando y donde estuviera disponible. Entonces se abrió campo en el mundo el concepto de solidaridad social y de la salud como un derecho humano fundamental. Los bienes y servicios que son fundamentales y necesarios para todos los miembros de una sociedad requieren de una intervención del Estado, que garantice su distribución universal y provea el subsidio para los que no pueden pagarlo. Es el mismo concepto que está a la base de la educación pública, de la seguridad, o de las vías de comunicación, para mencionar solo algunos ejemplos.

Les describiré ahora como he visto la reforma de la salud en los EE.UU. y lo que, en mi opinión, Colombia puede aprender de ella. Ha sido característica de nuestra idiosincrasia nacional, el pensar que todo lo que se hace en los países del mundo desarrollados, es mejor que lo que se hace en el nuestro. Sobre todo, y más que de cualquier otra parte, si viene de los EE.UU., que está a la cabeza de ese mundo. Como decía el poeta, "nuestro extranjerismo
Acta Med Colomb 2010; 35: 187-194.

*Conferencia Lombana Barreneche, dictada durante el XXI Congreso Colombiano de Medicina. Cali, 14 al 17 de octubre de 2010. Dr. Guillermo Vanegas Ramírez: MD, FACP, FCCP: ExPresidente ACMI 19982000, Miembro Emérito ACMI. Pulmonary and Critical Care Consultant, Alta Bates Summit Medical Center, Oakland, California. Práctica Privada en Neumología y Medicina de Sueño, Oakland, California. EE.UU.

E-mail: gvanegasmd@hotmail.com Recibido: 31/X/10 Aceptado: 11/XI/10 
es delicioso". Ciertamente estos países tienen y producen muchas cosas que merecen que las imitemos. Por algo son grandes y están adelante de nosotros, y de ellos tenemos mucho que aprender. Pero la reforma de la salud en los EE.UU. no es una de ellas. Indudablemente, la reforma mejoró notablemente el sistema de salud del país, en comparación con lo que había antes. Fue un paso hacia adelante y en la dirección correcta. Pero tímido e insuficiente. $Y$ en su mayor parte, inferior a lo que ya tenemos en Colombia, por lo menos en el papel. Algo tenemos que aprender de positivo, pero lo que más tenemos que aprender de esta reforma, es lo que no debemos imitar. Pero para no cometer errores y hacer cambios positivos en nuestro sistema, creo que vale la pena no solamente mirar lo que podemos aprender de la reciente experiencia americana, sino echar también un vistazo a lo que han hecho y lo que tienen otros países en este mismo campo.

Para entender mejor la reforma de la salud en los EE.UU., creo que hay que mirarla en la perspectiva de su evolución histórica y de la realidad en que tuvo lugar. La única reforma que el sistema de salud había tenido en los EE.UU. antes de esta última, tuvo lugar en 1965 durante la administración del presidente Lyndon Johnson y consistió en la fundación de Medicare y Medicaid, cuando el resto del mundo desarrollado, además de muchos otros países del llamado tercer mundo, habían ya aprobado leyes definiendo un sistema de seguridad social en salud 20 años antes, y habían obtenido significativos resultados. Hasta entonces, existían los seguros de salud privados, para el que los podía pagar, pero no había ningún sistema de seguridad social en salud. Esto no ha sido así porque los EE.UU. no hayan tenido las inteligencias, o los recursos humanos y económicos necesarios y suficientes para tener un sistema de seguridad social en salud a la par con el resto del mundo desarrollado, sino por diferencias sustanciales de filosofía política y económica. Este es uno de los campos en el que el sistema capitalista americano tiene su mayor expresión y diferencia con el resto de los países industrializados. Es la polaridad entre la oportunidad del negocio con grandes utilidades por un lado y la solidaridad social por el otro.

Veamos ahora cual era el esquema operacional del sistema de salud de los EE.UU. cuando se hizo la reforma de Obama. El seguro de salud no era obligatorio para nadie. Hasta los 65 años, los americanos tenían la opción de tener un seguro de salud privado, en la mayoría de los casos a través de su empleador, y los independientes por adquisición directa en el mercado. Solamente los empleados públicos, los miembros de las fuerzas armadas, los veteranos de guerra y los incapacitados tenían planes subsidiados por el Estado. No había un paquete básico de salud uniforme para todos los planes. Los seguros privados eran financiados conjuntamente por el empleado y el empleador, o en su totalidad por el independiente. Los planes privados estaban llenos de huecos y restricciones tales como preexistencias, limitaciones de cobertura, limitaciones con directorios médicos y listado de hospitales, limitaciones geográficas y topes en dólares por año y por vida. Las compañías aseguradoras tenían el derecho legal de negar o quitarle el seguro a cualquiera, en cualquier momento, por considerarlo de alto riesgo. A partir de los 65 años, se adquiría el derecho a Medicare, un plan nacional pagado por el Estado, financiado por impuestos obligatorios deducidos automáticamente de la nómina o ingreso durante toda la vida laboral. Medicare cubría solamente gastos de urgencias, hospitalización y cirugía, pero no cubría ningún servicio ambulatorio o consulta externa. A pesar de haber pagado impuestos durante toda la vida para la financiación de Medicare, y tener deducciones para su seguro privado, el usuario debía seguir pagando a Medicare una cuota mensual por el resto de su vida. Como la cobertura era insuficiente, el usuario tenía la opción de comprar una variedad de suplementos. O sea, doble tributación, de alto costo, con un solo servicio, y además lleno de huecos y limitaciones. Y existía Medicaid, el programa para los que estaban por debajo del nivel de pobreza absoluta. Su plan de beneficios era diferente, inferior, y mucho más limitado que el de Medicare. Era el equivalente de nuestro régimen subsidiado. Ninguno de estos planes cubría ningún servicio de medicina preventiva.

Este esquema dejaba un significativo sector de la población sin seguro de aproximadamente 45 millones de americanos; alrededor del 15\% de la población del país. Obviamente, cuando cualquiera de estos individuos presenta un problema de salud, o está en trabajo de parto, termina en un servicio de urgencias, que por ley tiene la obligación de atenderlo aunque no tenga seguro, incluyendo la hospitalización, cirugía o cuidado intensivo necesarios. Como es de esperarse, estos costos terminan asumiéndolos los usuarios de los planes privados.

Cuando el presidente Johnson presentó su proyecto de Medicare al Congreso, éste incluía, como su modelo canadiense, cobertura para todos los ciudadanos americanos desde su nacimiento hasta la muerte, sin preexistencias ni exclusiones, en competencia con los seguros privados. Pero los congresistas republicanos, con el argumento de que esto era socialismo, condicionaron sus votos a limitar la cobertura a los mayores de 65 años y a los incapacitados, con todas las limitaciones ya anotadas. El grito de "socialismo" fue entonces y ha seguido siendo suficiente en la gran democracia del norte para terminar cualquier debate sobre seguridad social, antes de que siquiera empiece. Tres presidentes antes de Johnson, dos más después de él y antes de Obama, habían intentado hacer una reforma de la salud y todos fracasaron en el intento, víctimas de la misma táctica.

El resultado de todo esto fue una increíble paradoja: el país más rico y a la vanguardia de la ciencia y la tecnología médica en el mundo, gasta más en salud que cualquier otro país, pero sus ciudadanos no reciben la atención que con ese gasto deberían tener. Efectivamente, en el escalafón hecho en el año 2000 por la Organización Mundial de la Salud de sus países miembros respecto a la calidad global de su salud 
pública, los EE.UU. estaban en el puesto 36, por debajo de Costa Rica y apenas por encima de Eslovenia. Colombia ocupó la posición 24 en ese escalafón. El único renglón en materia de salud pública en que EE.UU. ocupa el primer lugar en el mundo es el de gasto.

A la base de esta situación bastante extraña y paradójica, está una diferencia fundamental filosófica y política, como dije hace unos minutos. En los EE.UU., la salud no es considerada un derecho ciudadano sino una responsabilidad individual. Es la única democracia industrializada en cuya constitución política no se menciona la salud como un derecho ciudadano. En Colombia, este concepto se introdujo por primera vez en la constitución de 1991. La industria de la salud -como se le llama en EE.UU.- es precisamente eso: una industria millonaria que representa la sexta parte de su economía. En realidad, Estados Unidos no tiene un sistema nacional de salud, sino un mercado. Un gran mercado en el que la gente con dinero puede comprar servicios de excelente calidad y en el que mucha gente se queda sin acceso a los servicios cuando más los necesita.

El diseño del sistema de salud de cualquier país conlleva decisiones políticas, económicas y medicas. Pero la decisión fundamental es de orden moral, ético; y es la respuesta a esta simple pregunta:

¿Debe el Estado garantizar atención médica a todo el que la necesita?

Cualquiera que sea la respuesta para cada uno de los que, me escucha, pero sobre todo para los que respondemos SI, vale la pena revisar los sistemas de otros países para aprender ciertas lecciones que pueden ser útiles en el proceso de la revisión y reforma de la Ley 100. No se trata de hacer una copia Xerox del sistema de ningún país en particular, sino de tomar aquellos puntos y elementos que encontremos positivos, eliminar lo que encontremos negativo en el sistema que tenemos, y añadir, con las modificaciones que exija nuestra cultura diferente, lo que con una apreciación realista, nuestros recursos económicos y tecnológicos más limitados nos permitan implementar. Habiendo hecho las anteriores consideraciones, podemos entonces afirmar con una buena dosis de orgullo, satisfacción y optimismo hacia el futuro que, comparado con el sistema americano, el sistema nacional de salud de Colombia es globalmente superior. Aunque ciertamente tiene algunos defectos grandes e importantes, la Ley 100 en su mayor parte es un muy buen esquema. La triste realidad, sin embargo, es que los resultados no reflejan lo que se debería y podría esperar del diseño del sistema, porque algo pasó de la cocina a la mesa. Su implementación ha estado plagada de equivocaciones, distorsiones y graves omisiones.

El sistema de salud de cada país es un reflejo de su historia, de su política, de su economía, de su cultura y de sus valores nacionales. Hay diferentes modelos pero casi todos tienen los mismos principios.

Hay cuatro modelos básicos:

- El modelo Bismarck.
- El modelo Beveridge.

- El modelo de seguro nacional de salud.

- El modelo de medicina privada en el que cada quien paga de su bolsillo.

El modelo Bismarck, que toma su nombre del canciller prusiano Otto von Bismarck, quien lo inventó en el siglo XIX, se encuentra en Alemania, Francia, Bélgica, Suiza, Japón y en algunos países de América Latina. Bismarck fue en realidad el pionero de la seguridad social en salud en el mundo, y Alemania el primer país en implementarla. En este modelo, tanto los prestadores de servicios como los pagadores son entidades privadas. Los planes de salud son financiados conjuntamente por empleadores y empleados mediante deducciones obligatorias de los salarios. Estas entidades prestadoras de servicios son privadas, pero son todas sin ánimo de lucro y cubren toda la población. Los consultorios médicos y muchos hospitales son negocios privados, y el gobierno mantiene un control de costos mediante una estricta reglamentación de los servicios y honorarios médicos y de las tarifas de servicios hospitalarios.

El modelo Beveridge es el esquema básico del servicio nacional de salud de Inglaterra, que toma su nombre de su inspirador, William Henry Beveridge, un abogado, economista y audaz reformista social británico de la primera mitad del siglo XX. En este modelo, el servicio de salud es prestado por el Estado y financiado con impuestos de los contribuyentes. Los usuarios no reciben facturas por servicios médicos. La atención médica es un servicio público, igual a los bomberos, la policía o las bibliotecas públicas. La mayoría de los hospitales y clínicas son de propiedad del estado. Los médicos en estas instituciones son empleados públicos, pero también hay muchos otros que trabajan privadamente en sus consultorios, y reciben honorarios del Estado. Este esquema logra mantener costos per cápita bajos porque el Estado, como pagador único, controla lo que los médicos pueden hacer y lo que pueden cobrar. Además de Inglaterra, este sistema es usado en Hong Kong, Italia, España, Cuba y en la mayoría de los países escandinavos.

El modelo de seguro nacional de salud es básicamente una combinación entre el modelo Bismarck y el modelo Beveridge. Los prestadores de servicios de salud son privados, pero el pagador es un seguro nacional del Estado al que contribuyen todos los ciudadanos. Siendo un pagador único que cubre toda la población, el seguro nacional tiene un fuerte poder para negociar precios bajos. Además, el sistema define y limita los servicios que cubre y paga. El ejemplo paradigmático de este modelo es Canadá. Algunos de los países recientemente industrializados, o potencias emergentes, entre ellos Taiwán y Corea del Sur, han adoptado variaciones de este modelo.

El modelo de medicina privada con pago del bolsillo del usuario, es el que todavía se ve en algunos países del tercer mundo. Este esquema es extremamente simple y cruel: los ricos tienen acceso a servicio médico y los pobres pagan hasta donde pueden, o son atendidos de caridad, o se mueren. 
Esto es bastante común en áreas rurales de Africa, India, China y en algunos países de América Latina.

El sistema en los EE.UU., como lo habrán podido apreciar, es una colcha de retazos extremamente compleja que incluye todos estos modelos. La mayoría de los menores de 65 están en un modelo Bismarck. Los militares, veteranos y empleados del Estado tienen un modelo Beveridge, como el inglés. Para los mayores de 65 y los incapacitados, está Medicare, que es muy parecido al modelo del seguro nacional de Canadá. Y luego están los 45 millones que no tienen seguro y pagan de su bolsillo, o van a urgencias, o se mueren por no tener acceso al sistema.

Hasta la privatización del ISS, el sistema colombiano era una combinación del modelo Bismarck, de EPS e IPS privadas, y el modelo de seguro nacional de salud al estilo canadiense para los cubiertos por el ISS, y un sector de la población que no estaba cubierto por ningún seguro y dependía de su escaso bolsillo. Hoy día tiene un modelo Bismarck, con la importante diferencia de que casi todas las aseguradoras son con ánimo de lucro, más el sector de no asegurados que sigue pagando de su bolsillo o dependiendo de la caridad.

$\mathrm{Al}$ analizar y comparar sistemas de seguridad social en salud hay que considerar cuatro elementos:

- Cobertura.

- Calidad.

- Costos.

- Libertad de escoger.

\section{Cobertura}

Todas las democracias industrializadas y muchas de las naciones catalogadas como del segundo y tercer mundo, proveen cobertura universal. Es cuestión de justicia y equidad, además de conveniencia social y económica. Los EE.UU. son la más notable excepción. En estudios comparativos entre 23 países desarrollados, el Commonwealth Fund, una fundación privada americana dedicada a promover el mejoramiento de los sistemas de salud, clasificó a los EE.UU. en el último renglón en cuanto a acceso de su población a atención médica. Pero esto no es solamente un asunto de orden moral, sino también es un asunto de importancia práctica y económica. Los 45 millones sin seguro en EE.UU. son una considerable carga económica adicional al sistema, y muchos de ellos hacen parte de las 700000 familias que cada año se declaran en quiebra por no poder pagar cuantiosas cuentas hospitalarias según un estudio conjunto de las facultades de derecho y de medicina de la Universidad de Harvard publicado en 2005.

Otro renglón de muy importante consideración en la cobertura es el racionamiento de la atención médica: qué drogas, cuáles tratamientos o cirugías, o cuál tecnología es incluida en la cobertura. Racionamiento es para muchos una mala palabra y ciertamente lo es en los EE.UU. Pero la verdad es que todos los países racionan de una u otra manera, incluyendo los EE.UU. El racionamiento es indispensable para tener un sistema universal, eficaz, pero con costos manejables. Ningún país cuenta con recursos ilimitados y para su óptima utilización es imprescindible el racionamiento. La alternativa es la quiebra del sistema. Un ministro de salud de la Gran Bretaña recientemente definió el racionamiento en salud en su país así: "Nosotros cubrimos a todo el mundo, pero no cubrimos todo".

¿Debería el sistema cubrir los gastos necesarios para mantener vivo un paciente de 90 años con Alzheimer hasta que cumpla 91?. ¿Debería una mujer de 84 años debilitada e impedida con cáncer de seno, recibir la misma intensidad de tratamiento que otra con 44 años y sin ningún otro problema de salud fuera del cáncer?. ¿Debería el sistema cubrir Viagra?. ¿O Botox?.

Pero ¿quién decide qué es lo que debe estar cubierto? ¿Cómo se pueden aplicar esos límites con ecuanimidad? En los sistemas que ofrecen cobertura universal, estas decisiones las toma usualmente una agencia del Estado, totalmente apolítica, conformada por profesionales de varias disciplinas: médicos, economistas, sociólogos y expertos en bioética, que toma e implementa estas decisiones con criterios técnicos y médicos, basados en estudios comparativos de eficacia, seguridad y costo/beneficio, con total equidad y transparencia. En los EE.UU., este racionamiento lo hacen las compañías privadas de seguros, con criterios únicamente económicos para mejorar sus utilidades.

\section{Calidad}

Una medida bastante común de medir la calidad de un sistema de salud es la "mortalidad evitable" -o sea, qué tan bueno es un país en curar enfermedades curables. Otros parámetros de medirla son la mortalidad por enfermedades comunes y graves, la mortalidad infantil, la expectativa de vida, y mucho más importante que esta, la expectativa de vida saludable a partir de los 60 años, o sea, sobrevivencia con buena calidad de salud, sin dolencias incapacitantes, que es un excelente termómetro de la calidad de la salud pública de un país. Increíblemente, en estudios tanto de la OMS como del Commonwealth Fund, los EE.UU. clasifican de últimos entre las 23 primeras economías del mundo.

\section{Costo}

Ya sea que se mida como porcentaje del PIB o como gasto per cápita, los EE.UU. gastan en salud alrededor del doble de lo que gastan los demás países ricos: $17 \%$ de su PIB. Canadá y la mayoría de los países europeos gastan alrededor del 8 al 11\% y Colombia el 7.4\% de su PIB. ¿Cuál es la razón? Hay muchas razones, algunas más importantes que otras. Entre las más frecuentemente invocadas están: altos salarios y honorarios médicos, las drogas más caras del mundo, los costos de la educación médica y de los seguros de responsabilidad profesional y la medicina defensiva que estos generan. Pero aunque estos son factores que ciertamente contribuyen al alto costo de la salud en EE.UU., sumados representan menos de $10 \%$ del costo total. Entonces 
¿Cuál es la razón? La razón más importante es el esquema de funcionamiento de las aseguradoras y la complejidad laberíntica del sistema. Los EE.UU. son el único país desarrollado del mundo cuyo sistema de salud funciona total y exclusivamente con compañías aseguradoras privadas con ánimo de lucro. La mayoría de los economistas del mundo están de acuerdo en que esta es la forma más costosa de financiar un sistema de atención medica en cualquier país. Toda organización, pública o privada, con ánimo de lucro o sin él, tiene gastos administrativos. Hay la percepción muy común de que el sector privado puede manejar un sistema de salud con mayor eficiencia y menores costos que una burocracia estatal. Hay suficiente evidencia para probar lo contrario. La industria de aseguradoras privadas de salud en los EE.UU. tienen los costos administrativos más altos del sector en todo el mundo. Medicare, el programa creado por Johnson en 1965 y manejado por el Estado, tiene costos administrativos alrededor de 3\%, similar al que tiene el seguro nacional de salud en Canadá. Los costos administrativos del servicio nacional de salud de Inglaterra son de 5\%. El de las compañías aseguradoras privadas en EEU es de $16 \%$.

Como decíamos antes, la gran mayoría de los países miembros de las Naciones Unidas, definen la salud en su constitución como un derecho ciudadano fundamental. Sin embargo, como lo explican los abogados constitucionalistas, garantizar el derecho no es lo mismo que garantizar el servicio. Sobre todo cuando del dicho al hecho está el difícil trecho del costo que conlleva el subsidio. Todos los países desarrollados -menos los EE.UU., hasta antes de la reforma- garantizan la obligación de las compañías aseguradoras de vender seguro de salud a todo el mundo sin restricción alguna. Esto se conoce como "garantía de cobertura". Éstas argumentan -con razón- que tienen que seleccionar sus clientes, para evitar el problema conocido como "selección adversa" - término que se refiere a gente que decide no comprar seguro sino hasta que les diagnostican una enfermedad grave. De todos es sabido que la solución para la "selección adversa" es el "seguro obligatorio" para todos, ya sea privado, del gobierno, o subsidiado, y es una consecuencia necesaria de la "garantía de cobertura". Todos los países desarrollados tienen garantía de cobertura y seguro obligatorio. Los EE.UU. no tenían ninguno de los dos. La reforma empezó por introducir la obligatoriedad del seguro.

\section{Libertad de escoger}

Algunos sistemas de salud limitan las posibilidades del usuario de escoger: médicos, hospitales, tratamientos- para reducir costos. Esta es la característica de la mayor parte del sistema en los EE.UU. En países como Inglaterra, Francia, Alemania, Holanda y Japón, cualquier usuario del sistema puede, con su seguro, ir al médico y al hospital de su escogencia, en cualquier parte del país. Cualquier usuario puede escoger cualquiera de todos los planes en el mercado, y puede cambiar de plan cuantas veces quiera, dentro de un corto plazo. Los médicos pueden atender a cualquier paciente, independientemente de con cual aseguradora tiene su seguro. No hay listas limitadas o cerradas de médicos definidas y manejadas por las aseguradoras que les permiten disminuir gastos operacionales para aumentar sus ganancias manipulando los honorarios médicos. El paciente escoge, no la compañía aseguradora. El médico es independiente de las compañías y tiene oportunidad de trabajar con todos los pacientes de todas las compañías.

Hay muchas diferencias y opciones entre los diferentes sistemas y esquemas. No existe un sistema perfecto y aplicable a todos. Vivimos en una era tecnológica, y la tecnología tiene un rol principal en la medicina moderna. Todos se enfrentan al problema cada vez peor de la inflación de los costos. Esto ha llevado a que en todo momento haya uno o más países en el mundo embarcados en una nueva "reforma al sistema de salud", buscando casi siempre la forma de reducir los costos, manteniendo o mejorando la calidad, y sin negarle a la gente los beneficios de la medicina moderna. Sin embargo, esta búsqueda genera inevitablemente profundas y encendidas controversias. El economista americano TsungMei Cheng, profesor de economía en la Universidad de Harvard, ha formulado lo que con algo de humor y cinismo ha denominado las tres leyes universales de los sistemas de seguridad en salud:

1. No importa que tan bueno sea el sistema en cualquier país, sus habitantes siempre encontrarán de qué quejarse.

2. No importa cuánto dinero se invierta en salud, los hospitales y los médicos siempre dirán que no es suficiente.

3. Siempre se dice que la última reforma fue un fracaso o se quedó corta.

Al principio de la campaña presidencial de 2008 en los EE.UU., la inflación de los costos de salud estaba en ascenso y era más del doble de la inflación de la economía nacional. El gasto en salud se había trepado ya al 17\% del PIB.

La reforma de la salud y la guerra de Irak fueron los dos temas más importantes y de mayor controversia en la campaña. Barack Obama tomó como una de sus banderas principales la reforma del sistema de salud que definió como "ineficiente, extremamente costoso y quebrado," y que de seguir así, llevaría el país entero a la quiebra económica en menos de 50 años. Los demócratas querían un sistema de cobertura universal, con pagador único o con un seguro del Estado, llamado "opción pública”, que compitiera con las aseguradoras privadas, con mejores resultados de salud pública y con un gasto significativamente inferior. Los republicanos consideraban que los resultados en la calidad del servicio eran excelentes y por esa razón no se requerían cambios. De hecho, parte de su discurso repetido de campana era la definición del sistema de salud del país como "el mejor sistema de salud del mundo". No estaban muy interesados en ampliar la cobertura - ya que esto no se consideraba responsabilidad del Estado. Querían mantener al gobierno por fuera del sistema nacional de salud, porque ven su participación como una intromisión indeseable y perjudicial, y estaban de acuerdo sólo en la necesidad de reducir los costos 
por la amenaza que éstos representaban para la economía nacional. Además de estas diferencias, la gran discusión estaba en cómo reducir los costos. Pero a pesar de que los demócratas querían una ampliación de la cobertura, y tenían por consiguiente un elemento muy importante de filosofía y política social, la motivación de fondo, el propósito de la reforma y la presentación al electorado por ambos lados fue casi exclusivamente económica. El elemento moral de todo el asunto no hizo parte del debate. La inclusión de la cobertura universal, como elemento social, tenía un alto riesgo político ya que muchos americanos piensan que el tener seguro de salud es una responsabilidad personal y que los que tienen un ingreso suficiente y pagan impuestos, no tienen por qué subsidiar a los que no tienen los recursos para pagar un seguro privado.

Después de muchos esfuerzos y difíciles maniobras políticas para superar el bloqueo que el partido republicano montó en el Congreso, Obama pudo firmar en marzo de este año una ley de reforma al sistema de salud que en su versión final estaba aguada y empalidecida, pero que era de todas maneras mejor que lo que había antes, y sentaba las bases para continuar el proceso en el futuro.

Pero, ¿qué se logró, exactamente?

\section{Lo Malo}

La posibilidad de convertir el sistema en uno de pagador único no fue ni siquiera incluida en el debate y la propuesta de la "opción pública" murió rápidamente en el debate político ante el "lobbying" de las compañías aseguradoras para evitar la competencia. La totalidad de las compañías aseguradoras son privadas, la gran mayoría con ánimo de lucro. El sector privado mantiene su presencia en el mercado de los mayores de 65 con sus planes complementarios. Esto hará muy difícil la reducción del costo de las pólizas y de las primas para los usuarios y la disminución de los costos totales del sistema. La legislación que permite indemnizaciones millonarias por demandas de negligencia médica no tuvo por ahora ninguna modificación.

\section{Lo Bueno}

Los programas de Medicare y Medicaid no fueron eliminados ni disminuidos. El programa de Medicaid se modificará y extenderá para cubrir buena parte de los que hoy están sin seguro. El seguro de salud será obligatorio para todos. Habrá un paquete básico de servicios, igual para todas las pólizas que será establecido por el departamento de salud a nivel nacional. Las compañías aseguradoras no podrán negarle el seguro ni podrán quitarle la cobertura a nadie, a menos que se prueben casos de fraude. El valor de las primas será regulado por el gobierno. Se eliminaron las preexistencias al igual que los topes de cobertura en dólares por vida y por año por usuario. Se creó un "pool" o mercado especial de planes de precio reducido ofrecidos por las aseguradoras privadas, para los que no califiquen para Medicare o Medicaid y no tengan suficientes recursos para comprar seguro en el mercado privado. El sistema de remuneración a prestadores de pago por evento se modificará gradualmente a uno de pago por ejecución y resultados, incentivando la calidad y no la multiplicación de los servicios a expensas de la calidad y con mayores costos. Todas las pólizas tendrán que incluir cobertura para ciertos servicios de medicina preventiva. Todo el sector de seguros y de proveedores de servicios, que además de las aseguradoras incluye todos los hospitales, centros médicos y profesionales de la salud, tendrán que hacer la transición del papel a documentación electrónica tanto en el área administrativa como de historias clínicas, para aumentar la eficiencia, el intercambio y utilización de la información, y bajar los costos. Todos estos cambios tendrán una implementación gradual, con diferentes procesos y plazos, en los próximos ocho años, para evitar traumatismos y desestabilizaciones en el sistema. Si la implementación se hace de acuerdo con el plan estudiado, trazado y aprobado, habrá una significativa reducción en el gasto de salud que eliminará las quiebras de individuos y familias por gastos médicos en aproximadamente cuatro años, y contribuirá a eliminar el déficit fiscal del país en 10 años.

Sin embargo, a pesar de estos avances, todos parecen estar de acuerdo en que no se logrará la cobertura universal. Apenas tal vez se logrará aumentar de $85 \%$ a $90 \%$ o $93 \%$. La reducción de los costos tomará más de cuatro años, y muchos consideran que estas son cuentas alegres. Y para rematar, los republicanos están solo esperando retomar el control del Congreso en las elecciones del mes entrante para derogar la ley y volver al sistema anterior.

\section{¿Qué puede aprender Colombia de la reforma de salud en los EE.UU.?}

Lo primero es que, a pesar de las dificultades ideológicas y partidistas y las presiones de los grupos interesados en mantener el status quo, cuando hay voluntad política y se sigue una estrategia inteligente, se logran resultados. No siempre totales ni ideales, pero son pasos hacia adelante que hay que dar, con audacia y sin inhibiciones, cuando se quiere llegar a la meta, así sea a más largo plazo.

En mi opinión, lo único que el sistema americano tiene después de la reforma que es mejor que lo que hoy tiene Colombia es el programa de Medicare para los mayores de 65 años. Colombia lo tenía también en el ISS, no con la misma cobertura geográfica, pero sin limitación de edad. Creo que la eliminación o privatización del ISS fue un paso atrás en el proceso de mejoría del sistema nacional de salud. Hoy Colombia tiene un esquema todavía mejor que el de los EE.UU., pero entre las cosas que los dos sistemas tienen en común, están sus mayores fallas:

1) Ambos sistemas tienen dos niveles de servicio: contributivo y subsidiado, con acceso y planes de servicio diferentes, que resulta en la real eliminación de la equidad. Además de ser una injusta discriminación, hace extremamente difícil el control de costos, el control y mantenimiento de la calidad y la satisfacción de los usuarios.

2) Ambos sistemas operan el régimen contributivo con 
aseguradoras en su mayoría con ánimo de lucro. Estas características de ambos sistemas tienen un peso enorme en impedir los mejores resultados que ambos países deberían tener, proporciones guardadas de sus diferentes capacidades.

En Colombia hay consenso general en que se requiere una revisión y una reforma de la Ley 100. Sin embargo, creo que las reformas que la ley misma requiere son pocas. El mayor trabajo por hacer está en su reglamentación y en su implementación. Colombia funciona hoy con un sistema Bismarck: planes de salud ofrecidos en el mercado por empresas privadas prestadoras de servicios (las EPS), financiados conjuntamente por empleadores y empleados mediante deducciones obligatorias de la nómina. Los consultorios médicos y la mayoría de los hospitales y clínicas son negocios privados. A diferencia de los países en donde el modelo Bismarck ha sido exitoso, estas EPS son la mayoría con ánimo de lucro y no cubren toda la población sino la que puede pagar sus pólizas. Y tanto las primas de las mismas como los honorarios a los prestadores son definidos unilateralmente por ellas mismas. Las EPS tienen cuadros cerrados y limitados de médicos, que limitan al usuario en su capacidad de escoger y al médico en el libre ejercicio de su profesión. Este escenario es igual al que hay en los EE.UU. para el sector de la población con empleo y con un ingreso decente. En su conjunto, este es el sistema que produce los malos resultados que ya hemos revisado.

Pero Colombia no necesita cambiar de modelo, que sería mucho más complicado y costoso que seguir con el que tiene y que se adapta muy bien a su cultura, sus valores y costumbres, sino incluir aquellas características del modelo que han definido su éxito.

En mi opinión, se requieren tres cambios mayores:

1. Un solo paquete básico de beneficios para todos los usuarios. Un solo POS (Plan Obligatorio de Salud), bien definido en su cobertura, igual para los usuarios del régimen contributivo y los del régimen subsidiado.

2. La eliminación del lucro en la financiación del sistema.

3. La eliminación de los directorios médicos limitados en las EPSs.

Un mismo modelo para toda la población resulta en un sistema más simple, más eficiente, menos costoso y más justo. La definición del POS único implica que una institución del Estado, algo similar al NICE de Inglaterra (Instituto Nacional de Excelencia Clínica), conocido por todos ustedes, y cuyo equivalente en Colombia es el CRES (Comisión de Regulación en Salud), se encargue de su configuración y de su permanente revisión y actualización para poder hacer un racionamiento realista y humano que asegure la sostenibilidad del sistema. Y obviamente, la unificación del POS para los dos regímenes sería dándole al régimen subsidiado el nivel de beneficios que hoy tiene el contributivo, y no al revés.

La eliminación del lucro en la financiación no implica la desaparición de las EPS privadas. Por el contrario. Ellas hacen parte fundamental del modelo Bismarck. Privadas sí, pero sin ánimo de lucro. Esto, contrario a lo que piensan algunos, no elimina la competencia ni los incentivos. La competencia la hacen estas empresas para captar un mayor número de usuarios, que las hace más exitosas. Las "utilidades" se usan para pagar los salarios de los administradores, y para reinvertir en beneficios para los usuarios. No para repartir dividendos a accionistas o inversionistas. Las primas, así como los precios de los medicamentos, tienen que ser regulados, limitados y vigilados por un organismo del Estado. En este esquema puede haber o no una EPS pública. El propósito de que la haya debería ser crear un modelo de eficiencia y calidad de servicio para "arrastrar" a las EPS privadas por competencia en capturar usuarios. Sin embargo, esto implica un nivel de madurez política, depuración, eficiencia y transparencia en la burocracia oficial que no estoy seguro si ya lo ha alcanzado Colombia, pero debería ser una de sus mayores metas.

El seguro de salud que cubre el POS debe ser obligatorio para todos, como ya lo estipula la Ley 100, pero el cumplimiento de esta obligación debe ser real, sin excepciones ni alternativas. Esto requiere un sistema de vigilancia y verificación eficiente y sanciones con multas proporcionales al ingreso, que motiven fuertemente a todos a cumplir. Sin embargo, cuando se logran la universalidad, la equidad y la calidad, el mayor incentivo lo constituyen los beneficios y el no querer estar por fuera del sistema.

La remuneración de los prestadores, y específicamente de los médicos, es un elemento clave en la fluidez y la calidad de los servicios. Todo buen sistema de salud requiere que sus médicos sean bien pagados. Los médicos en Colombia están mal remunerados. Mientras esto no cambie, la eficiencia y la calidad estarán cojas.

Hay muchas experiencias y estudios en diferentes países que indican que el pago por ejecución y resultados, o inclusive el salario institucional, da mejores resultados para todos que el pago por evento. Se pueden considerar y ensayar diferentes opciones. Pero cualquiera que sea el sistema que se adopte, debe ser uno en cuya configuración y definición de las tarifas u honorarios, participe no sólo el pagador, sino también los usuarios, y por supuesto, el cuerpo médico. Debe pues ser el resultado de una deliberación periódica, racional y participativa que busque y llegue a un consenso aceptable y ojalá satisfactorio para todas las partes. No una imposición unilateral ventajosa por una de ellas.

Los directorios de médicos en las EPS deberían ser totalmente eliminados. El argumento de un listado de galenos prestigiosos para atraer clientes es engañoso. Sería mucho más atractivo poder ofrecer todo el cuerpo médico del país.

En la mayoría de los casos, una reforma no logra conseguir todos sus objetivos, y cuando se consiguen, con frecuencia no son duraderos, porque las circunstancias y los múltiples factores que definen y configuran el sistema cambian con el tiempo. Y por eso, más que una reforma, pueden ser necesarias varias reformas. Es un proceso casi en continuo, que requiere de una mente abierta al cambio y 
de mecanismos de revisión, vigilancia y adaptación.

Colombia tiene ahora una oportunidad histórica para reconfigurar su sistema de salud. El gobierno del presidente Santos ha sido claro en su propósito de hacerlo. El cuerpo médico tiene una responsabilidad con la sociedad y consigo mismo y debe, para cumplirla, comprometerse de lleno en ser un elemento de primera línea en ese proceso. La ACMI, en colaboración con la Federación Médica Colombiana, la Asociación Médica Colombiana, el Colegio Médico Colombiano y la Asociación Colombiana de Sociedades Científicas tiene ese reto. Yo sé que sus directivos lo han asumido y estoy seguro que harán un excelente trabajo.

Adelante !Buena vela y buena mar!. 